\title{
Poderão as estatinas provocar disfunção eréctil em homens adultos?
}

Ricardo Pinto Ribeiro, Isa Cavaleiro, Joana Jesus, Emília Salta

\begin{abstract}
RESUMO
Introdução: A disfunção eréctil (DE) é definida como uma incapacidade persistente de conseguir iniciar e manter uma erecção suficiente que permita ter uma performance sexual satisfatória. Esta pode ter uma etiologia neuropática, vascular, psicogénica ou endócrina, sendo vários os factores que contribuem ou agravam a DE. As estatinas, fármacos utilizados no tratamento das dislipidemias, poderão diminuir a produção de hormonas esteróides (por diminuição do colesterol, precursor da maioria das hormonas sexuais) e, por conseguinte, levar à diminuição dos níveis de testosterona, provocando assim disfunção eréctil.

Objectivo: Rever a evidência existente sobre o impacto das estatinas na função eréctil.

Metodologia: Pesquisa de revisões sistemáticas, meta-análises, ensaios clínicos controlados e aleatorizados e normas de orientação clínica publicados nas principais bases de dados (National Guidelines Clearinghouse, Guidelines Finder, CMA Infobase, The Cochrane Library, Bandolier, Embase e MEDLINE/PubMed), em Português, Inglês, Castelhano e Francês, com os termos MeSH Erectile Dysfunction AND Hydroxymethylglutaryl-CoA Reductase Inhibitors, no dia 23/05/2017. A avaliação da qualidade da evidência foi feita com recurso à escala SORT.

Critérios de inclusão: Estudos com indivíduos do sexo masculino com idade $\geq 18$ anos enquadrados nos termos de pesquisa. Da pesquisa foram excluídos todos os artigos anteriores à última revisão sistemática da literatura encontrada em cada base de dados. Resultados: Dos 52 artigos encontrados, após exclusão dos artigos que não se enquadravam no tema, artigos repetidos e artigos anteriores à última revisão sistemática da literatura em cada base de dados, três artigos cumpriam os critérios de inclusão: uma revisão sistemática e meta-análise, uma meta-análise e um artigo original).Apesar dos artigos serem heterogéneos, a maioria parece indicar que as estatinas poderão melhorar a DE ou reduzir o risco do seu aparecimento.

Conclusões: Da pesquisa efectuada conclui-se que as estatinas parecem diminuir sintomas de disfunção eréctil (força de recomendação B). Contudo, os estudos incluídos nas revisões sistemáticas e meta-análise analisadas são de curta duração e a maioria não avalia os níveis de testosterona no início e no fim do estudo, impedindo correlacionar a toma de estatinas com um hipogonadismo fármaco-induzido que poderia agravar ou manifestar a DE. Mais ensaios clínicos aleatorizados, com mais dados clínicos e laboratoriais e um maior tempo de follow-up serão necessários para melhor esclarecer os efeitos das estatinas na DE.
\end{abstract}

Palavras-chave: Disfunção eréctil; Estatinas; Inibidores da hidroximetilglutaril-CoA reductase; Hipogonadismo.

\section{INTRODUÇÃO}

A disfunção eréctil (DE) é definida como uma incapacidade persistente de conseguir iniciar e manter uma erecção suficiente que permita ter uma performance sexual satisfatória. ${ }^{1}$ É um problema de saúde frequente a nível mundial, estimando-se uma prevalência superior a 150 milhões de homens acima dos 40 anos. $^{2}$ A patofisiologia da DE é multifactorial e podem estar envolvidas causas arte-

Médicos Internos do $4^{\circ}$ ano de Medicina Geral e Familiar. USF Arco-Íris. riais, neurogénicas, hormonais, cavernosas, iatrogénicas e psicogénicas. ${ }^{1}$ É hoje comummente aceite que a DE poderá ser a consequência de uma doença vascular generalizada causada por uma disfunção endotelial. ${ }^{1-3}$ Nestes doentes a diminuição da produção de óxido nítrico $(\mathrm{ON})$ pelo endotélio vascular leva à diminuição da formação de monofosfato cíclico de guanosina, que é o segundo mensageiro que induz a vasodilatação arterial e corporal no corpo cavernoso. ${ }^{1}$ A doença aterosclerótica e a DE possuem factores de risco comuns, entre os quais diabetes mellitus, tabagismo, obesidade, 
hipertensão arterial, disfunção endotelial, assim como hipercolesterolémia. ${ }^{3}$

Os inibidores da fosfodiesterase tipo 5 (PDE5I), terapêutica de primeira linha da DE, impedem a PDE5 de degradar o monofosfato cíclico de guanosina. ${ }^{1}$

As estatinas (inibidores da enzima 3-hidroxi-metilglutaril-CoA reductase), agentes hipolipemiantes, são o tratamento de primeira linha para a dislipidemia associada às doenças cardiovasculares, reduzindo o risco de acidente vascular cerebral (AVC) e mortalidade ao atrasarem a progressão da aterosclerose..$^{2-3}$ As estatinas revertem a disfunção endotelial ao reduzirem a acção da lipoproteína de baixa densidade (LDL) oxidada nas células endoteliais, resultando num aumento da actividade do $\mathrm{ON} .{ }^{1,3} \mathrm{O}$ efeito benéfico destes fármacos sobre a função endotelial pode mesmo preceder a redução dos níveis de colesterol. ${ }^{1}$

Tendo em conta estas considerações, poder-se-á postular um efeito positivo das estatinas nos doentes com DE..$^{2-3}$

Contudo, existem relatos de aumento das taxas de incidência de DE entre os utilizadores de estatinas. ${ }^{2-3} \mathrm{Se}$ pensarmos que o colesterol é o precursor das hormonas esteróides e que as estatinas diminuem a síntese de colesterol de novo, podemos questionar se a terapêutica com estatinas poderá induzir um quadro de hipogonadismo e, consequentemente, levar ao aumento da disfunção eréctil. Um estudo recente constatou que os níveis de testosterona total e livre eram significativamente mais baixos nos doentes medicados com estatinas comparados com os que não faziam estatinas e que o uso de estatinas se associava a uma redução do volume testicular, assim como a uma maior prevalência de sinais e sintomas relacionados com hipogonadismo, pelo que se concluiu que a terapêutica com estatinas poderia induzir um hipogonadismo primário e ser considerada um factor de risco para DE. ${ }^{1}$

Esta revisão baseada na evidência tenta responder à questão: poderão as estatinas provocar disfunção eréctil?

\section{MÉTODOS}

Foi efectuada uma pesquisa de revisões sistemáticas (RS), meta-análises (MA), ensaios clínicos controlados e aleatorizados (ECA) e normas de orientação clínica publicados nas bases de dados National Guidelines Clearinghouse, Guidelines Finder, Canadian MedicalAs- sociation Infobase, The Cochrane Library, Bandolier, Embase e MEDLINE/PubMed, no dia 23/05/2017. Utilizaram-se os termos MeSH Erectile Dysfunction AND Hydroxymethylglutaryl-CoA Reductase Inhibitors. Foram seleccionados todos os artigos em Português, Inglês, Castelhano e Francês, desde a data de início das bases de dados até ao dia da pesquisa. Foram critérios de inclusão os estudos com indivíduos do sexo masculino com idade $\geq 18$ anos, que avaliassem a presença de disfunção eréctil em doentes medicados com estatinas. $\mathrm{O}$ outcome principal baseou-se na avaliação da diminuição ou aumento da taxa de prevalência de disfunção eréctil em doentes medicados com estatinas. Foram excluídos os estudos que não cumprissem os critérios de inclusão, por divergirem do objectivo do trabalho, por serem artigos repetidos ou estarem incluídos nas RS ou MA. A qualidade dos estudos e a força de recomendação foram avaliadas utilizando a escala de Strength of Recommendation Taxonomy (SORT), da American Family Physician.

\section{RESULTADOS}

Da pesquisa efectuada obtiveram-se 52 artigos. Destes, três preencheram os critérios de inclusão: uma revisão sistemática e meta-análise, uma meta-análise e um artigo original (Figura 1).

A MA de Kostis e colaboradores, ${ }^{3}$ publicada em 2014 (Quadro I), teve como principal objectivo avaliar o efeito do tratamento hipolipemiante com estatinas na disfunção eréctil, usando como referência a escala IIEF-5 (Five item version of the International Inventory on Erectil Function) (Quadro II).

Esta MA incluiu 11 ECA, dos quais sete compararam um grupo tratado com estatinas com um grupo tratado com placebo e quatro compararam o grupo tratado com estatinas com um grupo sem medicação. Foram ainda incluídos três estudos de coorte, incluídos apenas numa análise secundária dos resultados. Relativamente aos ECA, o tempo médio de follow-up foi de $4,2 \pm 1,8$ meses, abrangendo um total de 713 indivíduos com DE, com média de idade de 57,4 anos. Os três estudos de coorte abrangeram um total de 114 participantes com diagnóstico de DE, tendo sido comparada a sua pontuação na IIEF.

$\mathrm{Na}$ análise primária, que incluiu apenas os ECA, observou-se uma subida de 3,4 pontos na IIEF-5 (SD 0,8) 


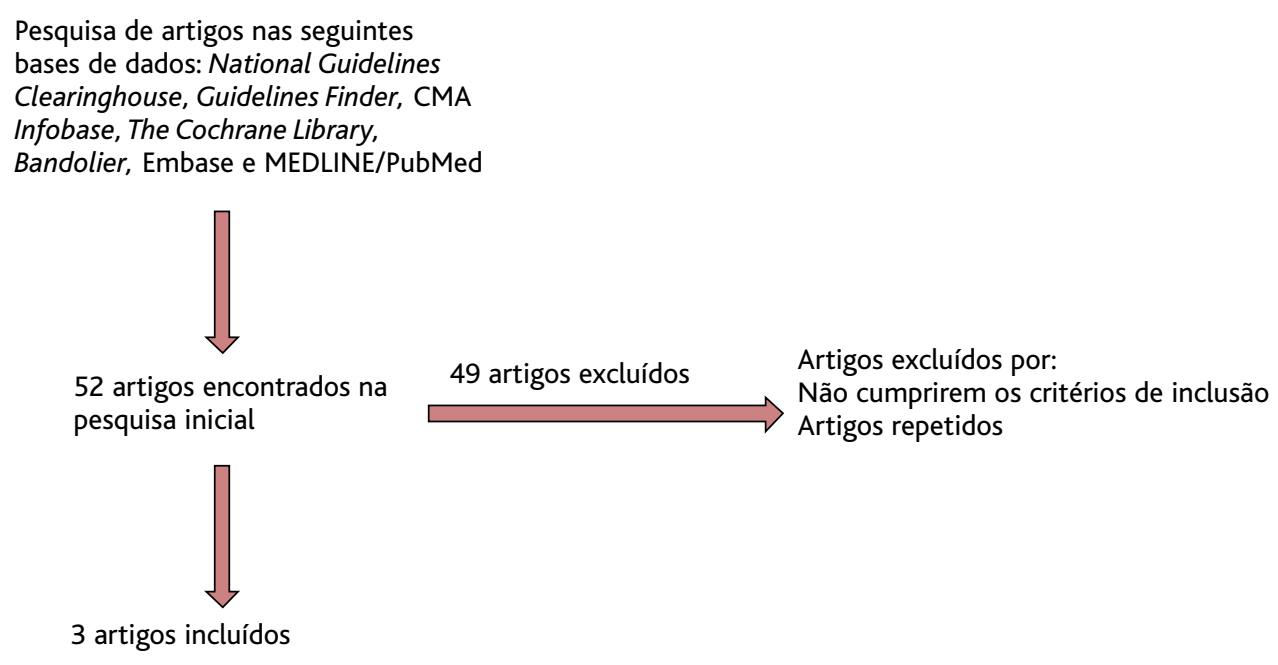

Figura 1. Organograma da selecção de artigos.

no grupo das estatinas relativamente ao grupo controlo, isto é, um efeito positivo das estatinas na DE.

A análise isolada dos três estudos de coorte não mostrou diferença significativa na variação da pontuação da IIEF antes e depois do tratamento $(0,2$ pontos, SD 4,4). No entanto, a análise de todos os estudos manteve a subida na IIEF, estatisticamente significativa, de 3,2 pontos (SD 0,8), ao analisar em conjunto o grupo controlo e de tratamento.

Esta MA apresenta como principais limitações o reduzido número de participantes e a curta duração de follow-up, o agrupar de estudos com diferentes subgrupos de participantes, a utilização de diferentes tipos de estatinas e metodologias distintas. No entanto, incluiu todos os ensaios clínicos randomizados publicados e os efeitos demonstrados mantêm-se consistentes, mesmo após múltiplas análises de sensibilidade. Deste modo, é-lhe atribuído um nível de evidência (NE) 2.

Cai e colaboradores seleccionaram sete ECAs que cumpriam os critérios de inclusão estabelecidos com o objectivo de avaliar o papel das estatinas no tratamento da DE. ${ }^{1}$ Os autores elaboraram uma meta-análise de cinco RCTs, que avaliou a resposta terapêutica às estatinas com recurso à escala IIEF, num total de 351 adultos do sexo masculino com queixas de DE, tendo ficado demonstrada uma melhoria da pontuação na escala (MD 3,27 pontos) após o tratamento com estatinas. No entanto, este trabalho apresentou várias limitações que podem ter enviesado os resultados, como o uso de estatinas diferentes, a heterogeneidade da duração da terapêutica, a subjectividade da escala IIEF e a utilização concomitante, e em diferentes regimes, de inibidores da fosfodiesterase-5. Deste modo, é-lhe atribuído um NE 2. Dois dos RCTs previamente seleccionados pelos autores não foram incluídos na meta-análise por terem utilizado questões diferentes da escala IIEF para avaliar a resposta ao fármaco. Os autores elaboraram também uma revisão sistemática dos sete ECAs seleccionados, sendo que cinco demonstraram uma melhoria das queixas de DE após a terapêutica com estatina, enquanto os outros dois não apresentaram diferenças significativas entre o grupo das estatinas e o grupo controlo. Deste modo, é-lhe atribuído um NE 2.

O estudo coorte retrospectivo de Chou e colaboradores, ${ }^{2}$ publicado em 2016 , teve como principal objectivo aferir a incidência de DE em indivíduos que tivessem sido medicados com estatinas, comparativamente com os que não iniciaram esta medicação. Para tal incluiu 39.272 indivíduos do sexo masculino com idades compreendidas entre os 40 e os 79 anos, os quais no período inicial do estudo não tinham diagnóstico de DE nem estavam sob tratamento com estatinas. O estudo baseou-se no período de Janeiro de 2000 até Dezembro de 2010. Os autores verificaram uma redução estatisticamente significativa do risco de incidência de DE na população medicada com estatinas ( $\mathrm{HR}=0,75)$, 


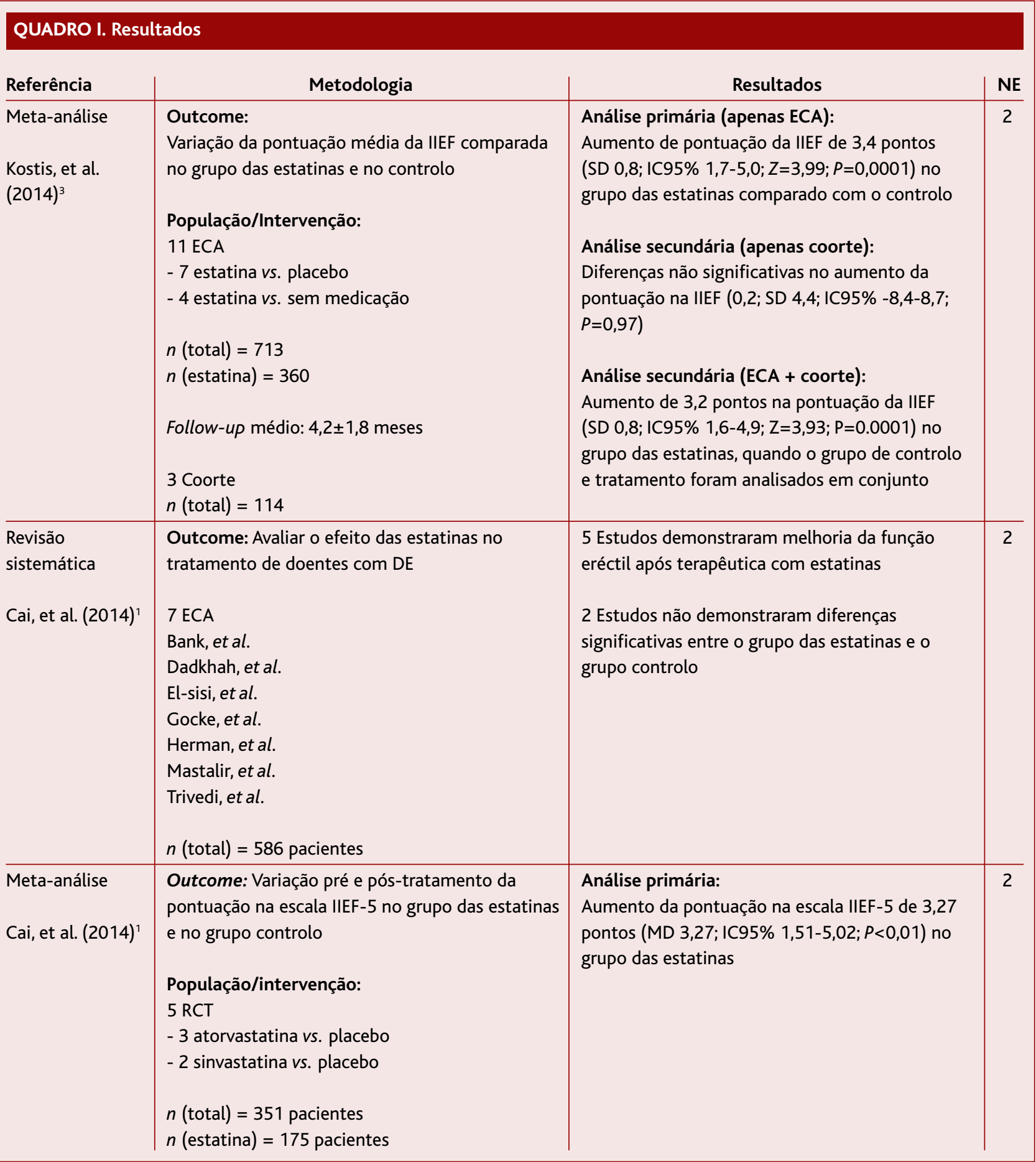

que se mostrou ainda mais impactante no caso da DE de causa orgânica $(\mathrm{HR}=0,70)$. $\mathrm{O}$ mesmo não se verificou para a DE psicogénica $(\mathrm{HR}=1,22)$. Quando analisados por faixa etária e por tipo de estatina observou-se um maior benefício do tratamento no grupo dos 40 aos 49 anos $(\mathrm{HR}=0,69)$, bem como nas estatinas de alta po- 


\section{QUADRO I. Resultados (continuação)}

\begin{tabular}{|c|c|c|c|}
\hline Referência & Metodologia & Resultados & NE \\
\hline $\begin{array}{l}\text { Estudo de coorte } \\
\text { retrospectivo } \\
\text { Chou, et al } \\
(2016)^{2}\end{array}$ & $\begin{array}{l}\text { Outcome: Avaliar a associação entre utilização de } \\
\text { estatinas e o risco de Incidência de DE } \\
\text { População: } \\
\text { Adultos do sexo masculino, entre } 40-79 \text { anos, } \\
\text { sem diagnóstico de DE e não medicados com } \\
\text { estatinas entre Jan. } 1997 \text { e Dez. } 1999 . \\
n \text { (total) }=39.272 \\
n \text { (estatina) }=9.818 \\
\text { Intervenção: } \\
\text { Estatinas + incidência de DE } \\
\text { vs. } \\
\text { Sem-estatinas + incidência de DE } \\
\text { Follow-up médio: } 4,19 \pm 3,00 \text { anos } \\
\text { (de Jan. } 2001 \text { até Dez. } 2010 \text { ou até à data de } \\
\text { ocorrência de DE diagnosticada por urologista) }\end{array}$ & 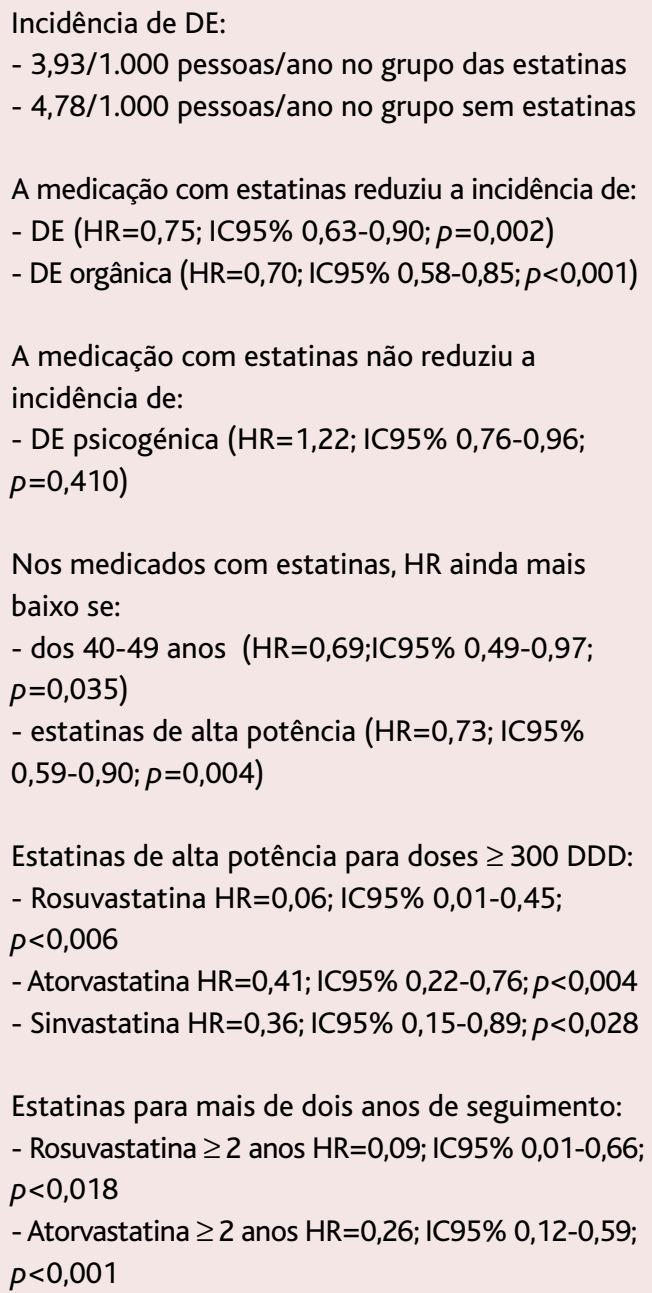 & 2 \\
\hline
\end{tabular}

Legenda: $\mathrm{NE}$ = nível de evidência.

tência $(\mathrm{HR}=0,73)$ : rosuvastatina, atorvastatina e sinvastatina.

Por outro lado, outros factores que estiveram associados a uma diminuição da incidência de $\mathrm{DE}$ foram doses mais elevadas das estatinas de alta potência na categoria de $\geq 300$ DDD e duração de tratamento igual ou superior a dois anos para rosuvastatina e atorvastatina.

Este artigo referiu como principais limitações o subdiagnóstico de DE (subjectividade da percepção individual do desempenho sexual, ausência de relato do problema ao médico, entre outros), ausência de informação clínica detalhada (escala IIEF e perfil lipídico), a ocorrência de confundimento pela indicação, ausência de informação clínica que não permitiu ajuste para alguns potenciais factores de confundimento, dados limitados à prescrição de estatinas (sem informação se estavam a ser tomadas actualmente e durante quanto tempo) e impossibilidade de avaliar o tempo entre o uso de estatina e o diagnóstico de DE. Deste modo, é-lhe atribuído um NE 2. 


\begin{tabular}{l}
$\begin{array}{l}\text { QUADRO II. Escala Índice Internacional de Função } \\
\text { Eréctil }^{5}\end{array}$ \\
Itens IIEF-5 - Versão portuguesa \\
\hline Nos últimos seis meses... \\
\hline 1. Como classifica a sua confiança em conseguir ter e \\
manter uma erecção? \\
\hline 2. Quando teve erecções com estimulação sexual, com que \\
frequência é que as suas erecções foram suficientemente \\
rígidas para conseguir a penetração? \\
3. Durante as relações sexuais, com que frequência foi capaz \\
de manter a sua erecção após a penetração? \\
4. Durante as relações sexuais, qual a dificuldade que teve \\
em manter a sua erecção até ao fim da relação sexual? \\
\hline $\begin{array}{l}\text { 5. Quando tentou ter relações sexuais, com que frequência } \\
\text { se sentiu satisfeito? }\end{array}$
\end{tabular}

Pontuação de 0-5 tipo Escala de Likert, com o grau de severidade dividido em cinco categorias: severo (5-7 pontos); moderado (8-11); suave a moderado (12-16); suave (17-21); não DE (22-25).

\section{CONCLUSÕES}

A evidência disponível é constituída por estudos que apresentam várias limitações importantes (seja o número de participantes, o follow-up, a heterogeneidade das metodologias, entre outros supracitados), pelo que lhe atribuímos uma força de recomendação B.

Ainda assim, os resultados obtidos são convergentes e apontam no sentido de existir um efeito benéfico das estatinas no tratamento da $\mathrm{DE}$, tanto na redução da sua incidência como na melhoria dos seus sintomas. As estatinas revertem a disfunção endotelial reduzindo a acção da lipoproteína de baixa densidade (LDL) oxidada nas células endoteliais, resultando assim num aumento da actividade do $\mathrm{ON} \mathrm{e}$, consequentemente, na indução da vasodilatação arterial e dos corpos cavernosos.

Deste modo, a questão inicialmente colocada pelos autores, segundo a qual a terapêutica com estatinas poderia ser causa de DE, ao reduzir os níveis de colesterol, principal precursor de testosterona, de acordo com a evidência actual, não parece verificar-se.
Contudo, os estudos analisados não avaliam os níveis de testosterona no início e no fim do estudo, impedindo correlacionar as estatinas com um hipogonadismo fármaco-induzido que poderia agravar ou manifestar a DE.

Para melhor esclarecer os efeitos das estatinas na DE, serão necessários mais ensaios clínicos aleatorizados, com mais dados clínicos e laboratoriais, assim como estudos de melhor qualidade e nível de evidência (sendo que todos os estudos incluídos nesta revisão apresentavam NE 2), permitindo a clínicos e pacientes uma tomada de decisão mais consistente e apoiada pela evidência científica.

\section{REFERÊNCIAS BIBLIOGRÁFICAS}

1. Cai X, Tian Y, Wu T, Cao CX, Bu SY, Wang KJ. The role of statins in erectile dysfunction: a systematic review and meta-analysis. Asian J Androl. 2014;16(3):461-6.

2. Chou CY, Yang YF, Chou YJ, Hu HY, Huang N. Statin use and incident erectile dysfunction: a nationwide propensity-matched cohort study in Taiwan. Int J Cardiol. 2016;202:883-8.

3. Kostis JB, Dobrzynski JM. The effect of statins on erectile dysfunction: a meta-analysis of randomized trials. J Sex Med. 2014;11(7):1626-35.

4. Ebell MH, Siwek J, Weiss BD, Woolf SH, Susman J, Ewigman B, et al. Strength of recommendation taxonomy (SORT): a patient-centered approach to grading evidence in the medical literature. Am Fam Physician. 2004;69(3):548-56.

5. Pechorro PS, Calvinho AM, Pereira NM, Vieira RX.Validação de uma versão portuguesa do Índice Internacional de Função Eréctil-5 (IIEF-5) [Validation of a Portuguese version of the International Index of Erectile Function-5 (IIEF-5)]. Rev Int Androl. 2011;9(1):3-9. Portuguese

\section{CONFLITO DE INTERESSES}

Os autores declaram não possuir quaisquer conflitos de interesse.

\section{NOTA FINAL}

Este artigo não foi redigido ao abrigo do novo acordo ortográfico por opção dos autores.

\section{ENDEREÇO PARA CORRESPONDÊNCIA}

Ricardo Pinto Ribeiro

E-mail: ricardopintoribeiro@gmail.com

https://orcid.org/0000-0002-4887-7288

Recebido em 29-04-2018

Aceite para publicação em 16-06-2019 


\section{ABSTRACT}

\section{CAN STATINS CAUSE ERECTILE DYSFUNCTION IN ADULT MEN?}

Introduction: Erectile dysfunction (ED) is defined as a persistent inability to initiate and maintain a sufficient erection to allow satisfactory sexual performance. This may have a neuropathic, vascular, psychogenic or endocrine etiology, and several factors contribute or aggravate ED. Statins, drugs used in the treatment of dyslipidemias, may decrease the production of steroid hormones (by lowering cholesterol, the precursor of most sex hormones) and therefore lead to lower testosterone levels, thus causing erectile dysfunction.

Objective: To review existing evidence on the impact of statins on erectile function.

Methodology: Research of systematic reviews, meta-analysis, randomized controlled clinical trials and clinical guidelines published in the main databases (National Guidelines Clearinghouse, Guidelines Finder, CMA Infobase, The Cochrane Library, Bandolier, Embase and MEDLINE/PubMed) in Portuguese, English, Spanish and French, with the terms MeSH 'Erectile Dysfunction' AND 'Hydroxymethylglutaryl-CoA Reductase Inhibitors' on 05/23/2017. The evaluation of the quality of the evidence was made using the SORT scale.

Inclusion Criteria: Studies with males aged $\geq 18$ years under the terms of the survey. The research excluded all articles previous to the last systematic review of the literature found in each database.

Results: Of the 52 articles found, after excluding articles that did not fit the theme, repeated articles and articles previous to the last systematic review of the literature in each database, three articles met the inclusion criteria: one systematic review and meta-analysis, one meta-analysis and one original article). Although the articles are heterogeneous, most seem to indicate that statins may improve ED or reduce the risk of ED.

Conclusions: From the research, we conclude that statins seem to decrease symptoms of erectile dysfunction (Strength of recommendation B). However, the studies included in the systematic reviews and meta-analysis analyzed are short-lived and most do not assess testosterone levels at the beginning and at the end of the study, preventing the correlation of statin intake with drug-induced hypogonadism that could aggravate or manifest the $D E$. Further randomized clinical trials with more clinical and laboratory data and a longer follow-up time will be needed to better elucidate the effects of statins in ED.

Keywords: Erectile dysfunction; Statins; Hydroxymethylglutaryl CoA reductase inhibitors; Hypogonadism. 